\title{
An ontological view of the human embryo. A paradigm
}

Carlos Alonso

Centro de Biología Molecular, CSIC-UAM, Facultad de Ciencias, Canto Blanco, 28049 Madrid, Spain

(Correspondence should be addressed to C Alonso; Email: Calonso@cbm.uam.es)

\begin{abstract}
In the present paper I analyze the existing paradigms explaining the ontological status of the organisms originating during a given developmental process and the potentiality these paradigms offer. These paradigms give us a hint of the ontological and ethical status of these organisms. It is described that during the history of human thought two theories have been developed to explain the origin of living organisms. One theory placed the world's origin and the origin of living organisms in a transcendent reality while the second stated that the growing complexity of these organisms was rooted in an internal 'dýnamis' (the internal potential of a thing, generally a living thing, to acquire new properties) that is present in each one of them. These paradigms are analysed concluding that at the time they were formulated there was no formal understanding of the process of development because the emergence of novelties through evolution was not included in them. At that time growth and development were two aspects of the same thing. A critical turning point in biological and philosophical thinking was provided by the founders of the DNA paradigm as the program directing development. Due to new insights, a paradigm is presented here which includes the epigenesic information as an essential element of the program of development together with the DNA. The consequence of this paradigm is that the program of development is time- and space-dependent and that the biological elements which originate during the process are not preformed but adopt specific values. This way of thinking introduces new approaches to the analysis of the value of the human embryo with repercussions in the ethical discussions on research programs using embryonic stem cells.
\end{abstract}

European Journal of Endocrinology 151 U17-U24

\section{Introduction}

In an article published in Science, John Ziman says that when he came into science the scientist rarely talked about ethical issues (1). He says that the reason was not because there were no such issues or that scientists were not, individually or in unofficial groups, speaking and acting about them. The reason was that ethics as such did not figure regularly in public discourse about science, in or beyond the scientific world (1). Nowadays, ethical issues are on the front page of newspapers and they promote discussions without precedent that frequently provoke violent ideological confrontations extending into the political arena. It is true that, in general, the assumptions that generate such discussions are based on dissenting situations and concepts and paradigms, but if we thoroughly examine the assumptions each one has we may find that they are not as different as they appear to be. The real problem is that the strategic positions previously taken do not allow the discussion to progress within rational frameworks and deliberative discussion pathways but rather they progress through a confused and monolithic situation frequently packed with insecurities and fears. On many occasions, moreover, the strategic positions are not supported by solid and consolidated observations.
Ever since mankind started to question the origin and evolution of the realities present in the Universe, it has been an obsession to elucidate the mechanisms that can explain the existence of living and non-living entities and the way the complexity of the phenomena we observe have arisen. Thus, throughout the history of human thought, there have been mainly two theories developed to explain the origin of living organisms and their complexity. One of the theories not only places the world's origin in a transcendent reality outside the observed ones but also claimed that the ontology of every single one of the realities observed should be explained by the intention that this transcendent reality had in mind when creating them: the world's observed entities depend on the existence of this transcendent reality and are sustained as what they are themselves, ontologically, by that transcendent being. The second alternative theory stated that the growing complexity of those observed entities is based on an internal 'dýnamis' (the internal potential of a thing, generally a living thing, to acquire new properties) that is present on each one of them. The world's entities are dependant, sustained and must be explained by themselves without the need for calling a transcendent reality from outside. 


\section{Morphogenesis of biological entities: two hypotheses}

In relation to morphogenesis of biological realities, particularly with regard to superior organisms, two extreme hypotheses have been postulated amongst a huge variety of ideas. All of the ideas seek to explain and give conceptual support to the existence of the enormous complexities that living organisms could acquire during the process of morphogenesis. All of these hypotheses had as a starting point an incontrovertible observation: some extraordinarily simple elements lead to the generation of some extraordinarily complex systems which occur as time passes. The important question is who or what guides the process: does the 'who' or the 'what' exist? The fact that from simple elements complex entities arise seems obvious because it happened. But how?

The explanatory paradigms for the origin of living creatures and particularly for the existence of mankind had to be formulated in two conceptual frameworks: (A) a substance existed, not translatable to physical terms, which contained in itself the complexity of the term which acted as an outstanding element of the generation process, or (B) the existence of an element should be postulated, translatable to physical terms, which would be able to direct the generative process of complexity. In this latter case it would be necessary to postulate that the simple element was simple only in appearance because it had to contain the complexity. Both theories invoked an outstanding element governing the process. The difference between the two theories lies in the fact that while in one of them the 'who' or the 'what' was not different from the material reality, in the other one the 'who' or the 'what' had to be situated inside the material reality but without being of the same nature. Philosophically, the need for the existence of the 'who' or the 'what' of a different nature from the matter that directed the process of morphogenesis had to be postulated and understood since simple matter could not have given rise to the complexity of existing elements. Thus, the words 'matter' and 'material' were coined to discriminate between the 'director' of the process and the 'substrate' of the process itself. The 'material' element was above matter but was not the spirit. What was it? Thus, the two theories diverged radically in the nature of the element governing the process of change. If the element (vital principle) programming the processes belonged to the individual living creature without physical identification with it, there was a need for postulating that the vital principle was of material nature but not matter. In this situation the material element could not be identified with the term matter, from which it originates.

In this hypothesis, the idea of the existence of an element governing the process of development being the source of the complexity did not seem to present any conceptual problem. The material element (not matter) governing the process had the intrinsic capacity of directing the process of the generation of the complexity. The complexity was, even though as a potency, in the material element. The 'form', as proposed by Aristotle (2), or the vital principle in the case of animated beings, held that capacity. The vital principle held the complexity of the final organism before its existence, although in another way only as a potency. Because of that the vital principle is its efficient and final cause.

For the theory based on the hypothesis of the matter as holding a 'dýnamis', which was also matter in nature, a serious problem was raised: it could not be conceived how something which was not complex in itself or something having a low complexity in structure could lead to another reality of a higher order of complexity. This was one of the reasons why for such a long time the nucleic acids were rejected as being the element governing development. Even less was it possible to understand how the appearance of complex beings from simple ones could happen.

The problem lay in the fact that there was no concept or paradigm able to explain the transformation phenomenon and the source of new entities with ontological qualities which did not exist in time prior to their actual existence. Thus, many authors chose the idea that in the original element, the complexity of the term was already and always present but in a non-revealed way. The interactions, mainly with the environment, explained the appearance of the individual characteristics of the term. Thus, the origin must have contained, in some way, the term to which it led through development. The concept of progressive growth could be understood and presented in formal terms. The appearance of a new complex phenomenon non-existent on the origin, through the process of transformation, could not be formally rationalized.

The most suitable concept was to propose that realities of great complexity were nothing but the phenomenal actualisation of what the entity was in the origin. So, the generation process was nothing but the continuous unravelling of the content of the original element giving rise to the complexity of the term. In fact, then, there was no real distinction between growth and development. That is why the only existing difference between a given complex structure and the simple one from which originated, was only apparent. There was no substantial difference between them. The complex structures were potentially contained in the original. The potential held by the original, nonetheless, was something else apart from a 'pure being able to be' because it contained the final organism in potency. In this assumption, the complex end was not just 'being able to be' but the updated version of what already was. The simple was the potency of the complex.

Would there exist any material element containing the map of the entity which is the final term of the generation? In the case of not finding it, it seemed obvious to 
postulate the existence of a factor, outside the physicochemical material, untranslatable to those physical terms, holding the capacity of directing the process of development. Otherwise, we would have to accept proposals close to the theory of the homunculus which stated that humankind and each human being or organism was contained inside the spermatozoon head. Only during their embryological history would the content be revealed. But the content was already there from the beginning. Otherwise, it would be necessary to appeal for the hypothesis of the existence of a 'vital principle' of material origin but not matter. The hypothesis of the homunculus was completely suitable and agreed with the existing concepts of development. It was logical, then, to give to the zygote the name of real embryo and to give to the embryo the value of the term.

The tension between the followers of spiritualism and the promoters of the material 'dynamism' was served. In such a case, there was no other alternative but to clarify the conflicting situation and to look for the entity governing the developmental processes. The identification of such an entity would draw the discussion away from the dead end which it had reached. Otherwise, the position maintained by both parties was so distant that they were involved in a sterile discussion using different languages. It did not seem possible to design experiments to prove the existence of the spirit or the 'vital principle'. Experiments would have to be designed to prove the existence of the material 'dynamism', but under the condition that it should contain the complexity of the end.

Since a concept explaining the emergence of ontologically new biological entities from some others which are simple in constitution is not easily intelligible, it appears that practically there is no difference conceptually between what the philosophers said in the IV century $\mathrm{BC}$ and what has been written by the most notorious embryologist of the XVI century. The authors of that period understood that living creatures breed from one another. Through the embryological period, the multi-cellular organisms do not transform from simple ones but they are bred. Even Darwin himself thought that the spermatozoa should contain emissions from the whole adult organs shaped in particles or gemmules that, by transmission, will lead to the formation of cells with the same or similar characteristics of the organisms they originated or proceeded from.

\section{DNA as a carrier of information for development}

A crucial turning point, apart from the traditional biological paradigms used up to that time, was given by Oswall Schmeideberg in 1896 (3) when he declared that inheritance should have chemical support. Particularly important was the proposition of Mazia, in 1952, stating that DNA 'did nothing, but was only used as a simple mould for the synthesis of the molecules' (4). Boivin et al. concluded that DNA acts as a 'code' (5). For the first time it was suggested that the cellular processes are carried out through the decoding of the information contained within a chemical molecule. However, all of these proposals were constantly rejected because the simple could not be the origin of the complex. So, in spite of the existence of reasons favouring nucleic acids as the genetic depository of inheritance, and because of reasons difficult to understand, the belief that nucleic acids constituted the inheritance material was constantly rejected. How could an apparently inactive molecule, not having the characteristics of the proteins, provide a biological function as specific as that that was required for the constitution of complex organisms and with the potential for governing development and to be the reference from which complex systems are formed?

The work of the biologist Boivin and of the chemist Chargaff and their colleagues (6-9) and of those from the school of the physical chemists Wilkins, Franklin and Pauling (10-14) prepared the way for the proposal of Watson and Crick (15). In fact, Chargaff spoke about DNA being a molecule with nearly infinite possibilities of containing different information in its base sequence. Finally, Watson and Crick, in their famous work, stated that it has not escaped our notice that the specific pairing we have postulated immediately suggests a possible copying mechanism for the genetic material'. For some a dogma was born: DNA codifies, RNA transports the information read in the DNA, proteins are synthesized from this information and the organisms are built from these molecules. In fact, the entire developmental process could be reduced to the construction of a building from a map. The complexity emerges from the assembling of the coded elements. DNA was turned into the element being the ruler of morphogenesis.

The interesting concept in this context is that the molecule (DNA) directing development is not material as in the 'vital principle' but it is matter by nature. We could say that the 'dýnamis' had been discovered but with a different ontology. The end-terminus was, as in an image, contained in the origin and was constructed from elements resulting from decoding of the information contained in the DNA. Thus, the term is an up-to-date constitution of something which originated as a potency by synthesis of what was already an archetype in the DNA. The informative capacity of the DNA was then seen to be an essential element of the developmental program. By reading this program and its execution the new living creature is constituted. The complexity of the end-terminus was foreshadowed in the original element. The end-terminus was not contained in the original element, as was postulated by the homunculus hypothesis, but it was present as an image in one of the units of the cell, the nucleic acids. 
Although with a more appropriate formulation to experimental scientific discoveries, the DNA was converted, for some, into a molecular homunculus. This concept lent DNA some properties that were never claimed by the founders of the DNA paradigm or by later researchers. This new concept, substituting the necessity of invoking the vital principle to rule the developmental process, was a good starting point for some people as a way to understand molecularly the mechanisms of development: the DNA contained the essential elements to create a new living creature and to design it. Thus the origin, for the simple reason that the DNA contained the information to build the term, already had, as a map and in a potential form, the characteristics of the term. It was 'real potentia' of the term. In this framework, the DNA was the efficient and the final cause of the term.

Nowadays, in maintaining the informative character of DNA as a mould for protein synthesis a new current of opinion is being created, as yet restricted, that postulates that the process of morphogenesis is developed through a construction process and, even more appropriately, a constitution of novelties. Various types of information interplay and intervene for the constitution of the end-terminus. Not all types of information signals are codified in the DNA molecule. The new non-codified information emerges from temporary interaction between cells and molecules. These information signals are needed to establish the directionality of every single stage of the process. The information is contained within the net of epistatic, epigenesic and epigenetic events occurring during the process of development. This network of events, rather than the DNA, together with cellular interaction and the DNA constitute the developmental program. Thus, the program of development includes the DNA but it is not limited to the genome, as indicated by Strohman, Brenner and colleagues (16-18).

The real problem for the proper understanding of this new concept lies, according to John Maddox, in being able to understand the nature of the information contained in a molecular gradient flux and in the temporary interaction between molecules and cells (19-23). It was already difficult to understand that a chemical molecule could contain the information necessary to codify thousands of different molecules. Even now it is really difficult to understand how some information can be contained in the temporality of a molecular flux or in cellular interactions. We do not have a formal paradigm to understand how this may happen, but we have no choice but to accept this fact, especially when we know that small differences in gradients of the same molecule or molecules can contain pieces of information which are qualitatively different. In fact, there is a lack of formal understanding of the rules that allow the cell to design big and robust informative nets.

\section{Molecular and cellular interactions as carriers of information for development}

Thus, in biomedicine a new paradigm is being generated proposing that genetic inheritance is not the only inheritance. Something else apart from genes is inherited. Dynamic cellular structures are also inherited. They provide strength and robustness to the system. Specifically, through germinal cells we inherit structures that favour, together with DNA, the starting signal for successive auto-organisation. This information is of an epigenetic pleiotrophic kind. In this new paradigm several types of information are postulated. (i) The existence of signals emerging from the interaction between molecules codified in the DNA, (ii) the existence of information coming from the interaction of molecules not codified in the DNA and (iii) the existence of gradients of inorganic molecules that can function as morphogens. On top of that, the information coming from cellular interactions is paramount. It is from the dynamics of the whole cell, and not from the separated elements, that the biological information unity, as a program, is constituted. However, because the informative signals are originated in time and they are specific of the space-time in which cellular groups converge, they do not exist prior to their emergence.

John Maddox (19-23) suggests that a conceptual frame must be generated including quantitative methods to examine concentration flux of molecules directing gene expression control. In this paradigm, the DNA is just a reference system, where, in a request format, information for the generation of protein molecules is contained. This information is needed for the formation of the elements that form the cellular structures and for those needed for the construction of non-protein molecules. All these types of molecules (information) are needed equally to properly establish the molecular dialogue between molecules and cells. The strong and specific cellular structures and contexts interpret the various instances in which a gene can be transcribed and be functional. Thus, information above the gene level has to be postulated (24). However, given the numerous ways in which a single piece of information can be interpreted and that the cells are the biological units modulating the interpretation of the genes, it is the intermolecular dialogue together with the DNA that is one of the important elements constituting the temporal program from which a new transforming information emerges during development. Thus, the program of development is not static but it is generated as it happens.

This new concept can be summarised by the proposal of Strohman when he says that genetic determinism for complex characters has assumed the notion of a genetic program to explain the casual union between genes and phenotype. But it has been found that this assumption has no experimental verification. There are no genetic programs. There are merely genes codifying for 
proteins. Some of these genes and their products are very important. For example, some products join to DNA and are involved in regulation of morphological pattern development. When these genes mutate or do not exist at all, they generate deep changes in the pattern character. But these genes also exist in an epigenetic context and are conditioned by this context for the control of their expression in a species-specific way $(16,17)$. Thus, development is coded in emerging molecules and ionic fluxes and decoded as they are carried out and contained in the developing organism as a whole. The emergent and resulting information from molecular interactions direct the generation of further developmental stages $(25,26)$.

As early as 1961, Waddington indicated that 'the morphogenetic phenomenon brings us face to face with the fact that we have to find an explanation independent of cell properties in an individual way but with well operating factors interacting either inside each cell or between cellular groups $(27,28)$. That is why he postulated that even in the case of cellular interactions communications that could be analytically reduced to gene activation it could easily be proven that they are from another complex nature. The most relevant conclusion is that these types of information do not take place until the interacting systems are present. It is obvious that the development of an individual is carried out through a functional process in continuity in which the transforming operations take place on a given biological substrate. During the process there is no rupture, at any time, of the individual genetic or biological entity or basic structure as a whole. This fact, however, does not imply that during the process biological novelties do not appear which at the same time transform the biological entity which is the substrate of the information. If this is so, not all the novelties have the same ontological value.

From the time of the discovery of the programmed apoptotic phenomenon (cell death), the necessity of invoking cellular interactions for the construction of homeostatic complex systems became increasingly clear. Apoptosis controls proliferation and determination (migration), proliferation and differentiation (choosing cellular destiny), proliferation without differentiation (renovation), survival and death, and, as far as we know for the moment, it is essential for the synaptogenesis among neurones and the selection for the repertoire of lymphocyte receptors. Apoptosis is a physiological process essential for normal development to take place during embryo metamorphosis. Then, apoptosis is a paradigmatic example of the epistatic and epigenesic phenomena of temporary nature. While its execution is dependent on gene expression, the starting point of that process, in precise time and space, is dependant on the niche. This means that the process is activated or suppressed by signals proceeding from molecular gradients, either from other cells or from the environment, but always temporally depen- dent. Cellular apoptosis only occurs in a context determining it to happen or not to happen. A failure in the context can lead to fatal consequences for the organism. The genetic information informs the process. The niche determines the when and the how of its execution. This temporally dependent information which determines whether to start or not to start the process is not programmed in the DNA but in the net of cellular and molecular interactions.

\section{Foetus-mother cross-talk as carrier of information for development}

Among placental organisms there exists another source of information that has drastic repercussions in the process of early development and also has a determining and transforming character with regard to the embryo - the mother. It seems normal that up to a few decades ago, and even for some people now, it was thought that external codifications to the embryo having transforming capacity did not exist. The molecular understanding we had at that time about mother-embryo interactions came from the embryology of oviparous organisms. In the ovum of oviparous organisms the necessary and sufficient information for development resides in the DNA and in the cellular architecture. Nowadays we know that in placental organisms this is not the case. The mother not only provides the embryo with the nutrients necessary for development but also provides molecular signalling directing the differential expression of genes essential for a normal development process. Thus, at least in the early stages, the mother belongs to the developmental program of the fetus. This fact by no means indicates that the fetus belongs to the mother's biological system. It means that the biological system of the fetus cannot sustain itself without the biological entity of the mother which gives information to the developing system, particularly in the early stages. This is an event that has emerged during the evolution of the systems.

A paradigmatic example (given actual knowledge) of epigenesic information coming from the mother (symbiotic), essential for qualitative development and efficient formation of the nervous system during embryonic stage, is the function of the maternal hormone thyroxine $\left(\mathrm{T}_{4}\right)$. This transference has been extensively confirmed in the rat system of development $(29-35)$. This hormone is transferred from the mother to the embryo through the placenta at a certain stage of the development of the embryo and is detected in the embryo before the embryo can express its own hormone. Since this hormone regulates gene expression at a level that is essential for correct embryo development, the information provided by the mother's hormone must belong to the program of embryo development. In the embryo there exists, at the moment of $\mathrm{T}_{4}$ transfer, protein receptors 
that interact with the hormone. Biologically speaking, it does not seem to be congruent that receptors for the hormone are present in the embryo when there is no production of the hormone, particularly as this hormone is necessary for the proper development of the nervous system. The presence of these receptors is only sensible biologically if the hormone is available from other sources. If motherly $\mathrm{T}_{4}$ does not exist or is at a low concentration at the precise moment of development, the constitution of the embryo's nervous system is deficient and abnormal. For this reason the external information should be understood as being an integral part of the embryo's developmental program.

In principle, there is no reason to believe that a similar type of information transfer from the mother to the embryo does not occur in other cases, particularly during the first stages of development. During implantation there is an intense dialogue between the mother and the embryo that allows activation of certain embryonic genes. This interplay allows development to be carried out and to occur. If this series of interactions are correct it is congruent to think that amongst placental organisms evolution may have eliminated some signalling that the mother can provide to the embryo during development, in a space-temporary way. This signalling would be part of the embryo's development program. In the first steps of development mother and embryo constitute a complex system made up of two different individual organisms, but in symbiosis. One of the individuals is in formation, the other one is formed. That is the way it is strengthened - in the first stages of development there exists a constant transfer of molecular information between the element supporting development and the one that is being generated.

All of these concepts are pushing forward a change in paradigm which postulates that during development the organisms are generated inside an evolutionary process in which there is the emergence of entities which are qualitatively new. The new emerging elements possess their own ontological entity having new informative capacity for further development of the substrate. Obviously this emerging entity is linked to the preceding and posterior realities occurring during the process since, as indicated above, the genetic individual substrate upon which transformations are carried out is not interrupted at any time. But this fact does not force us to maintain that all the entities succeeding at the time, because they constitute the substrate upon which transformations occur, are qualitatively the same.

In modern times, in order to defend the ontological statute of the early embryo as a biological reality associated with the person, the concept of development as a process having functional continuous totality within the entire period has been stressed. That is to say that the process is carried out without interruptions into a sequence of functions with a 'prior' and a 'posterior' state. Because it is continuous, the process is a totality. That is to say, each stage must be understood in the context of the whole. Obviously, this concept is correct only if we do not include in it what it should be proven, that is: that the 'prior' has identical value as the 'posterior'. Because the development process is a functional continuum, it is a totality without qualitatively different parts. Then, from the concept that the organism is a functional continuum it would be easy to deduce that all stages must participate from a common, and the same, value. However, it is difficult to see any biological argument or philosophical concept that could unambiguously manifest the existence of any element in the origin defining the totality of the term. The idea that the developmental process is a functional continuum at best indicates that if the final organism is a person, the origin must participate from the value of the term. But, there is no reason to attest that the biological entity defining the origin has to be valuably identified with the final organism.

\section{Concluding remarks}

Personally, I do not understand and comprehend the reasons why while agreeing with the essence of the 'process philosophy' in the context of the ontogeny of living organisms from embryos, it is necessary to demand that all the elements of a process should have the same value either ethically or ontologically. Obviously, different elements of a process cannot be understood without any reference to a 'before' and an 'after'. That is why all the participating stages have a common value. But participation does not mean identity unless the origin contains the totality and is, in some way, the efficient and final cause of the term. From a biological point of view, a proposition of this kind cannot be made because it is based on an incomplete paradigm. It is obvious that the moment the ovum is fertilised by the spermatozoon defines a 'before' and an 'after' in embryonic development with regard to the genetic and epigenetic constitution of the ovum and the spermatozoon. The ovum has a given cellular and genetic architecture. The sperm has its own genetic and cellular architecture. The zygote has a polarised and genetic cellular architecture different from the previous cells. However, it has to be proven that the moment called 'afterwards' or post-fertilisation has such an entity that it defines the moment of personhood, or terminal, constitution.

From all that has been said it is obvious that the zygote is a human being and an individual because it has the characteristics of something having ontology and of being biologically human and individual. But we must not confuse being, which is an ontological attribute, with person, which is also an ontological attribute but qualified with ownership. The human zygote is human because it belongs to the biologically human species. However, not every biological entity belonging to the human species is a human-person. 
The human zygote is an individual because the zygote is indiviso in se et diviso at qualibet alio. But not every individual because of 'being something', and undivided in act and biologically human, is a person. Following the concepts developed by Ricardo de San Victor (36) we could say that the early embryo 'is' a nature while the person 'has' a nature. The person is a subject that owns something, its ontology. The embryo is a nature but it does not own a nature. From my point of view, the human embryo is not a subject holding and having nature. We could state that the embryo is only nature. Obviously the nature also has value although different from the subject holding and having a nature.

One of the consequences of this paradigm is that in the zygote there is not the final stamp of the terminal phenotype because an isomorphic map cannot be established between the genetic products and the complex phenotype. The zygote cannot be conceived as a cell containing the whole of the development program constituting the terminal phenotype. Even more, I am not sure if in this context the zygote could be described, with propriety, as an embryo as such, since it has no intrinsic and autonomous capacity of being the end. Only in a temporal view can the embryo be called an embryo. Both characteristics are necessary and sine qua non to be able to attribute to the embryo the term potency. I think it would be more appropriate to define the zygote, and in particular the one generated in vitro, as a cell open to the generation of an individual and a human being. It has the characteristics to do so when it is confronted with some given information because it has the capacity of responding to the information from within and from the niche - the mother. Here the zygote is dignified because it is a cell holding unique characteristics - to generate a person. The mother is also symbiotically dignified as she ensures that such characteristics manifest themselves in a personal human reality. If a mouse embryonic stem cell is placed in a tetra-haploid blastocyst, an organism is produced that is genetically identical to the original stem cell. Would it be possible to say, then, that the stem cell was an embryo of the generated organism?.

Undoubtedly, a change in the paradigm of how embryonic transformations are carried out and the possibility of the emergence of ontological novelties during development is going to have huge implications in determining the value that should be conferred on particular stages of the organisms during development. In this paradigm, as in any other paradigm, the problem lies in being able to decide when the system is constituted. From my point of view and because of the arguments I have presented previously it cannot be stated that the person is constituted either in the zygote stage or in any particular early stage of embryonic development.

We have to be conscious that none of the arguments I have indicated up to now can be used to determine in an apodictic way that the embryo, in its early stages, has not the ethical value attributable to the person. To declare the opposite would be a biological and philosophical mistake given that the concept of personhood cannot be affirmed or denied following biological arguments. The arguments given are valid, however, to question the reasons adopted in the past and even in the present, to assert that the embryo has the ethical value of a person.

Would it not be much more true to say that the terminal entity - the human person - is only achieved when the terminal entity has gone through the space and time period of its auto-organisation? Would it not be much more congruent to assimilate the constitution with a biological period in which the system has acquired stability, strength and robustness in such a way that the 'attractors' or external factors cannot modify substantially the developing organism? In negative terms: would it be more congruent with the person's dignity not to identify dignity with a stage so unstable as the early stage of development where nearly $80 \%$ of the zygotes starting development are naturally removed because of the lack of proper timeand space-defined information? Zubiri defines reality as a structured field or a close structure of elements or notes that, when present, establish the constitution of the organism (33). When this structure is coherent the field reaches constitutional sufficiency, and therefore, substantiveness. Since, for Zubiri, if there is no constitutional sufficiency there is no reality, something not having constitutional sufficiency could not be the object of its own rights nor the object of outside duties (33). The zygote has its own constitutional sufficiency to be a zygote but the question of whether or not the zygote may have constitutional sufficiency to have the value of the terminus (person), which is the real subject to whom values are undoubtedly and inexcusably attributed, cannot be excluded.

In societies with barely any changes, ethical challenges are minimal. The behaviour is fixed. In a society involved in a frenetic change, like the actual one, the ethical challenge is permanent, in addition to being urgent, because the answers previously given are not valid. Furthermore, in a plural society the answers have to be plural if we want them to be accepted. However, the plurality of ethics is altogether a different subject. Thomas Kung points out that if we are imbued with the parameters dictated by a particular paradigm, many years of work are needed to discover the possible internal weakness that can be embedded in the paradigm before new perspectives can emerge (34). If there is no evidence against it, it is practically impossible to abandon the paradigm in which we are working. I believe that there are arguments that indicate that there is a new paradigm to understand the process of development of biological realities which can illuminate the kind of ethical values we may attribute to them. Evolution and the appearance of novelties into a given process enter into the paradigm. Thus, in a 
given process these qualitatively new novelties may adopt different values.

\section{Acknowledgements}

This work was supported by grant Bio2002-04049C01 from CICYT.

\section{References}

1 Ziman JM. Why must scientists become more ethically sensitive than they used to be? Science $1998 \mathbf{2 8 2} 1813-1814$.

2 Aristotle. Reproducción de los animales II 1-2-3.

3 Schmeideberg O. Physiologisch - chemische untersuchungen über die lachsmilch. Archives fur Experimentelle Pathologie und Pharmakologie 189637100.

4 Mazia D. Physiology of the cell nucleus. In Modern Trends in Physiology and Biochemistry, pp 77-122. New York: Barron, E.S.C., 1952.

5 Boivin A, Vendrely R \& Vendrely C. Biochemie de l'heredité. Du acide desoxyribonucleique du noyan cellulaire, depositaire des caractères héréditaires; arguments d'ordre analytique. Comptes Rendus Hebdomadaires des Seances de l'Academie des Sciences 1948 $2261061-1064$.

6 Chargaff E \& Shapiro HS. Remarks on sequence characteristics of the DNA and transfer RNA of yeast. PNAS $196859161-163$.

7 Rudner R, Karkas JD \& Chargaff E. Separation of B. subtilis DNA into complementary strands. I Biological properties. PNAS 1968 $60630-635$.

8 Chargaff E. Preface to a grammar of biology. A hundred years of nucleic acid research. Science $1971172637-642$.

9 Chargaff E. Chemical specificity of nucleic acids and mechanism of their enzymatic degradation. 1950. Experientia $1994 \mathbf{5 0}$ 368-376.

10 Wilkins MH, Strokes AR \& Wilson HR. Molecular structure of deoxypentose nucleic acids. 1953. Nature $2003 \mathbf{4 2 1} 398-400$ discussion 396.

11 Pauling L \& Corey RB. Structure of the nucleic acids. Nature 1953 171346.

12 Pauling L. Molecular basis of biological specificity. Nature 1974 $248769-771$.

13 Franklin SE \& Gosling RG. Molecular configuration in sodium thymonucleate. Nature 1953171 740-741.

14 Franklin RE \& Gosling RG. Molecular configuration in sodium thymonucleate. 1953. Nature $2003 \mathbf{4 2 1} 400-401$, discussion 396.

15 Watson JD \& Crick FH. Molecular structure of nucleic acids; a structure for deoxyribose nucleic acid. Nature $1953 \quad \mathbf{1 7 1}$ $737-738$.

16 Strohman R. Epigenesis: the missing beat in biotechnology. Biotechnology $199412156-164$

17 Brenner S, Dov W, Hewrskowitz L \& Thomas R. Genes and development: molecular and logical themes. Genetics $1990 \mathbf{1 2 6}$ 479-486.
18 Strohman R. Thermodynamics: old laws in medicine and complex disease. Nature Biotechnology 200321 477-479.

19 Maddox J. The mechanics of life. Nature 1994372 33-34.

20 Maddox J. Watson, Crick and the future of DNA. Nature 1993362 105.

21 Maddox J. Long-range correlations within DNA. Nature 1992358 103.

22 Maddox J. Finding wood among the trees. Nature 198833311

23 Maddox J. Is molecular biology yet a science? Nature 1992355 201.

24 Quesada I, Martin F, Roche E \& Soria B. Nutrients induce different $\mathrm{Ca}(2+)$ signals in cytosol and nucleus in pancreatic beta-cells. Diabetes 200453 (Suppl 1) S92-S95.

25 Strohman R. Five stages of the human genome project. Nature Biotechnology 199917112.

26 Strohman RC. The coming Kuhnian revolution in biology. Nature Biotechnology 199715 194-200.

27 Waddington $\mathrm{CH}$. Molecular biology or ultrastructural biology? Naturwissenschaften 1961190184.

28 Waddington CH. The Pattern of Genetic Development. New York: Columbia University Press, 1961.

29 Morreale de Escobar G. Maternal hypothyroxinemia versus hypothyroidism and potential neurodevelopmental. Alterations of her offspring. Annales d'Endocrinologie $20036 \mathbf{6 4} 51-52$.

30 Lavado-Autric R, Auso E, Garcia-Velasco JV, Arufe Mdel C, Escobar del Rey F, Berbel P \& Morreale de Escobar G. Early maternal hypothyroxinemia alters histogenesis and cerebral cortex cytoarchitecture of the progeny. Journal of Clinical Investigation 2003111 1073-1082.

31 Calvo RM, Jauniaux E, Gulbis B, Asuncion M, Gervy C, Contempre B \& Morreale de Escobar G. Fetal tissues are exposed to biologically relevant free thyroxine concentrations during early phases of development. Journal of Clinical Endocrinology and Metabolism $2002871768-1777$.

32 Morreale de Escobar G \& Escobar del Rey F. Maternal thyroid deficiency during pregnancy and subsequent neuropsychological development of the child. New England Journal of Medicine 1999 341 2015-2016; author reply 2017.

33 Morreale de Escobar G, Obregon MJ, Calvo R \& Escobar del Rey F. Effects of iodine deficiency on thyroid hormone metabolism and the brain in fetal rats: the role of the maternal transfer of thyroxine. American Journal of Clinical Nutrition 199357 (Suppl 2) 280S-285S.

34 Calvo R, Obregon MJ, Escobar del Rey F \& Morreale de Escobar G. The rat placenta and the transfer of thyroid hormones from the mother to the fetus. Effects of maternal thyroid status. Endocrinology $1992131357-365$.

35 Kohler J. Transfer and metabolism of thyroid gland hormones in the placenta. Acta Medica Austriaca 199724 138-143.

36 Ricardo de san Victor. De Trinitate VI 23.

37 Zubiri X. El hombre y Dios. Madrid: Alianza, 1984.

38 Kuhn T. The Structure of Scientific Revolutions. Chicago: University of Chicago Press, 1962.

Received 18 May 2004

Accepted 5 August 2004 